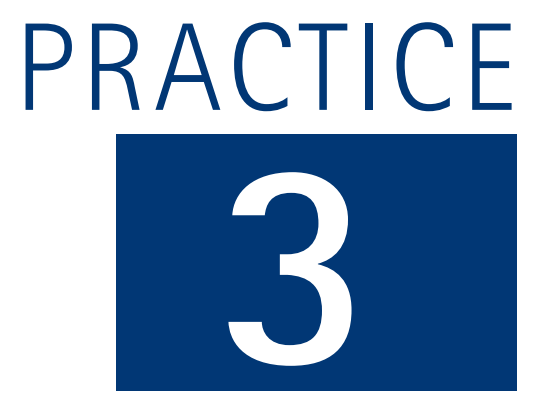

\title{
Treatment planning of implants in the aesthetic zone
}

\author{
S. Jivraj ${ }^{1}$ and W. Chee ${ }^{2}$
}

Aesthetic restoration of anterior teeth with implant supported restorations is one of the most difficult procedures to execute. Bone resorption following anterior tooth extraction often compromises gingival tissue levels for the implant restoration. In the last 10 years the focus has shifted from osseointegration, to creation of an implant borne restoration which is in harmony with the surrounding hard and soft tissue. Complete reconstruction of tooth and gingival related aesthetics remains the primary objective and in some instances can be very difficult to achieve.

\begin{tabular}{|c|}
\hline IMPLANTS \\
\hline 1. Rationale for dental implants \\
\hline $\begin{array}{l}\text { 2. Treatment planning of implants in } \\
\text { posterior quadrants }\end{array}$ \\
\hline $\begin{array}{l}\text { 3. Treatment planning of implants } \\
\text { in the aesthetic zone }\end{array}$ \\
\hline $\begin{array}{l}\text { 4. Surgical guidelines for dental } \\
\text { implant placement }\end{array}$ \\
\hline $\begin{array}{l}\text { 5. Immediate implant placement: } \\
\text { treatment planning and surgical } \\
\text { steps for successful outcomes }\end{array}$ \\
\hline $\begin{array}{l}\text { 6. Treatment planning of the } \\
\text { edentulous maxilla }\end{array}$ \\
\hline $\begin{array}{l}\text { 7. Treatment planning of the } \\
\text { edentulous mandible }\end{array}$ \\
\hline $\begin{array}{l}\text { 8. Impressions techniques for } \\
\text { implant dentistry }\end{array}$ \\
\hline $\begin{array}{l}\text { 9. Screw versus cemented implant } \\
\text { supported restorations }\end{array}$ \\
\hline $\begin{array}{l}\text { 10. Designing abutments for } \\
\text { cement retained implant supported } \\
\text { restorations }\end{array}$ \\
\hline 11. Connecting implants to teeth \\
\hline $\begin{array}{l}\text { 12. Transitioning a patient from } \\
\text { teeth to implants }\end{array}$ \\
\hline $\begin{array}{l}\text { 13. The role of orthodontics in } \\
\text { implant dentistry }\end{array}$ \\
\hline $\begin{array}{l}\text { 14. Interdisciplinary approach to } \\
\text { implant dentistry }\end{array}$ \\
\hline $\begin{array}{l}\text { 15. Factors that affect individual } \\
\text { tooth prognosis and choices in } \\
\text { contemporary treatment planning }\end{array}$ \\
\hline 16. Maintenance and failures \\
\hline
\end{tabular}

The predictability of aesthetic success depends on the tissue loss present at the initiation of treatment. The greater the amount of bone and soft tissue loss, the more difficult it becomes to produce an ideal aesthetic result. Single tooth implants have a high degree of predictability as the adjacent teeth can provide the morphological substructure that is required to restore natural gingival and papillary architecture. Replacement of multiple missing teeth in the aesthetic zone is challenging particularly when the three dimensional architecture of the existing bone and soft tissue is deficient. The bony housing in this instance would require augmentation to provide a configuration that permits placement of implants in optimal positions which in turn would result in pleasing aesthetics. The purpose of this article is to look at the diagnostic factors that affect the predictability of peri-implant aesthetics. Emphasis is placed on those parameters which are critical to overall treatment planning.

$1^{*}$ Chairman, Section of Fixed Prosthodontics and Operative Dentistry, University of Southern California School of Dentistry/ Private

Prosthodontics Practitioner, Sherman Oaks and Torrance California; ${ }^{2}$ Ralph Prosthodontics Practitioner, Sherman Oaks and Torrance California; ${ }^{2}$ Ralph
W. and Jean L. Bleak Professor of Restorative Dentistry, Director of Implant W. and Jean L. Bleak Professor of Restorative Dentistry, Director of Implant
Dentistry at the University of Southern California School of Dentistry I Private Prosthodontics Practitioner, Pasadena, California

*Correspondence to: Dr Sajid Jivraj, School of Dentistry, Rm. 4372 University Park, University of Southern California, Los Angeles, CA 90089-0641, USA Email:jivraj@usc.edu

Refereed Paper

๑) British Dental Journal 2006; 201 : 77-89

DOI: $10.1038 /$ sj.bdj.4813820
Marketing enquiries have identified aesthetics as one of the major reasons why dentists use dental implants in their surgeries. If we go by that premise it is reasonable to speculate that the aesthetic results provided with dental implants should be similar to the aesthetics provided with more conventional modes of therapy such as fixed and removable partial dentures. However, achieving aesthetics with implant restorations is significantly more challenging than that with conventional restorations. Diagnosis and appropriate treatment planning are critical in obtaining a successful outcome. Many manufacturers will identify their systems as aesthetic; from an objective perspective components in themselves are not aesthetic. There is not a single component available from a manufacturer which would be the ideal replacement for a maxillary central incisor. Aesthetic outcomes are based on many variables. It is not the specific implant design, surface characteristics or type of abutment that will guarantee an aesthetic result. It is rather the time spent on data collection in reaching a correct diagnosis that pays dividends in terms of function and aesthetics. ${ }^{1}$

Root form cylindrical implants placed following surgical techniques described by Branemark et al. have proven to be a predictable method for anchoring replacement teeth to the jaw bone. ${ }^{2,3}$ Today clinicians can prescribe the use of implants with the knowledge and confidence that they will predictably integrate into the jaw 
bone. The successful integration of an implant, however, is not sufficient to declare success; implants placed in poor restorative positions result in unaesthetic restorations that provide little satisfaction for the clinician or the patient (Figs 1-3). The above illustrations demonstrate the complexity of implant use in aesthetic zones and the importance of proper treatment planning prior to implant placement.

Providing an aesthetic outcome requires understanding of the objective and subjective criteria related to hard and soft tissue aesthetics. ${ }^{4}$ Both dental and gingival aesthetics act together to provide a smile with harmony and balance. The clinician must be aware of parameters related to gingival morphology, form and dimension, characterisation, surface texture and colour ${ }^{5}$ (Fig. 4). Ceramists can often produce restorations to match adjacent teeth in terms of colour, however if the surrounding tissues are not reconstructed an aesthetic outcome is not likely (Fig. 5). The ultimate aim is for the implant restoration to harmonise with the frame of the smile, face and more importantly the individual.

Treatment planning must address hard and soft tissue deficiencies and combine this with precision in implant placement. Only with this approach can implant restorations be indistinguishable from the adjacent teeth (Fig. 6).

Recreating what nature provided can be a formidable challenge. The physiology of wound healing after tooth extraction creates an unfavourable soft tissue complex. The remaining mucosa often recedes palatally and apically. Often this results in a restoration that appears long and this is compounded by the absence of interdental papilla (Fig. 7).

The predictability of the aesthetic outcome of an implant restoration is dependent on many variables including but not limited to the following:

1) Patient selection and smile line

2) Tooth position

3) Root position of the adjacent teeth

4) Biotype of the periodontium and tooth shape

5) The bony anatomy of the implant site

6) The position of the implant.

\section{Patient selection and smile line}

Patients who are candidates for replacement of an anterior tooth with an implant supported restoration must understand the benefits of an implant restoration. They must also understand the additional length of time required for treatment and additional costs that will be incurred. The clinician must also understand the patient's desires. In most cases the patient's primary demand is an aesthetic tooth replacement; with this in mind it is important to establish sound clinical concepts with clearly defined parameters that lead to successful aesthetics with long term stability of the peri-implant tissues.

The major indication for a single tooth implant restoration is preservation (non preparation) of one or more of the adjacent teeth (Fig. 8), and reduction in the rate of alveolar resorption. Additional indications would be restoration of a missing tooth to maintain a diastema and preservation of extensive fixed restorations that are intact.

A patient's aesthetic expectations must also be evaluated together with their lip activity and lip length. In an average smile 75-100\% of the maxillary incisors and the interproximal gingiva are displayed. In a high smile line additional gingival tissue is exposed. Less than 75\% of the incisors are exposed in a low smile line (Figs 9-11). ${ }^{6}$ The clinician should be aware that the patient who presents with unacceptable tooth health, shade or position may not give a full smile when asked. Previous photographs may aid in determining the natural position of the patient's lip when smiling.

A high smile line poses considerable challenges when planning for implant supported restorations in the aesthetic zone because the restoration and gingival tissues are completely displayed. In these types of clinical situations maximal efforts towards maintaining periimplant tissue support throughout the planning, provisional, surgical and restorative phases will be required. An article later in the series will discuss soft tissue management with provisional restorations.

The low smile line is a less critical situation because the implant restoration interface which will be hidden behind the upper lip. However this cannot be assumed and the patient's input must be sought to confirm this. 'The aesthetic zone is where the patient thinks it is.'

\section{Tooth position}

The tooth needs to be evaluated in three planes of space: apicocoronal, faciolingual and mesiodistal. The existing tooth position will significantly influence the presenting gingival architecture. In many instances teeth with a poor prognosis are thoughtlessly extracted. These teeth can significantly influence both the hard and soft tissue configuration.

Apico-coronal On assessment of the apico-coronal position of the tooth it may be more apical, more coronal or ideal and mimic the level of the adjacent gingival margin (Fig. 12). Numerous authors have shown that following extraction and insertion of an ovate pontic there is likely to be up to $2 \mathrm{~mm}$ of gingival recession, and on extraction and placement of an implant immediately the migration of the gingival margin is likely to approximate $1 \mathrm{~mm} .^{7,8}$

The implications this has from a practical perspective are that if there is a hopeless tooth positioned ideally or apically and this is extracted, the gingival margin is likely to migrate apically. Restoratively, long clinical crowns, pink porcelain or visible metal margins will result, compromising the aesthetic outcome. These teeth can benefit from orthodontic extrusion (Fig. 13) prior to extraction which will serve to position the gingival level at a more harmonious level. ${ }^{9,10}$ 
Facio-lingual In this dimension the tooth position may present with different concerns. The tooth may be positioned too far facially; this often results in very thin or non existent labial bone. These teeth are not good candidates for orthodontic extrusion because of inadequate underlying bone. Extraction of these teeth results in significant vertical bone loss and collapse of the gingival architecture. This type of situation would benefit from bone augmentation procedures prior to implant placement. A tooth positioned more lingually would benefit from the presence of an increased amount of facial bone. This situation is more favourable prior to extraction since the resultant discrepancy in the facial free gingival margin may be minimal. ${ }^{9}$

Mesio-distal The proximity of the adjacent teeth necessary to provide proximal support and volume of interdental papillae should be evaluated. Ideally the mesiodistal tooth width should be equal to that of the contra lateral tooth so that an aesthetic outcome can be achieved (Figs 14-15). Excess or deficiencies in this dimension should be addressed through the use of orthodontics, enameloplasty or restorations. For patients with diastemas it is imperative that the decision to maintain or close the space be made prior to implant placement. If the patient refuses the above options to close the space and insists on closing the space with the implant restoration there is a likelihood that a black triangle may ensue. This results from inadequate support from the adjacent tooth to maintain the papilla. It is important that the clinician discusses this with the patient ahead of time so disappointment with the final outcome is avoided (Figs 16-17).

\section{Root position of the adjacent teeth}

Part of the diagnostic work up for patients who need implants is a peri-apical radiograph, as often root position will preclude placing of implants. Many of these patients can benefit from orthodontics to reposition malposed teeth. If the patient illustrated in Figures 18 and 19 desired implant restorations to replace congenitally missing maxillary lateral incisors, orthodontic therapy would be necessary to move the roots of the cuspid and central incisor to allow room for ideal implant placement.

Teeth with root proximity also possess very little interproximal bone; this thin bone creates a greater risk of lateral resorption which will decrease the vertical bone height after extraction or implant placement. When teeth are present the use of orthodontics serves as a valuable adjunct to create space. This can be advantageous for support of proximal gingival architecture. ${ }^{11,12}$

\section{Biotype of periodontium and tooth shape}

The position of the gingival tissue around a tooth is determined by the connective tissue attachment and by the bone level. Two differ- ent periodontal biotypes have been described in relation to the morphology of the interdental papilla and the osseous architecture: the thin scalloped periodontium and the thick flat periodontium. ${ }^{13}$

The thin scalloped periodontium found in less than $15 \%$ of cases is characterised by a delicate soft tissue curtain, a scalloped underlying osseous form and often has dehiscence and fenestrations and a reduced quantity and quality of keratinised mucosa. Generally interproximal tissue does not completely fill the space between adjacent teeth. This form of gingiva reacts to insults by receding facially and interproximally. As recession occurs and the inter-root bone resorbs, the subsequent soft tissue loss compromises the overall aesthetic result (Fig. 20).

The tooth form in this type exhibits a contact point towards the incisal third essentially triangular anatomic crowns and contact areas of teeth that are small facio-lingually and apicocoronally. Due to extreme taper of the roots the bone interproximally tends to be thicker.

Characteristics of the soft tissue biotype will play a prominent role in final planning for the shoulder position of the implant. A thin biotype with highly scalloped tissue will require the implant body and shoulder to be placed more palatal to mask any titanium show through. When implants are placed toward the palate a slightly deeper placement is required to allow for proper emergence profile.

Combining previous factors in a patient with a high lip line and a thin biotype is extremely difficult to treat. Patients who fit into these treatment categories should be made aware of the challenges involved in obtaining an aesthetic result before treatment begins.

The thick flat periodontal biotype is characterised by a denser more fibrotic soft tissue curtain, a flat thicker underlying osseous form and an increased quantity and quality of attached keratinised gingiva. This tissue often reacts to insults by pocket formation. Flat gingiva is associated with a tooth form that is more bulbous. Contact areas are located more toward the middle third of the tooth; primarily square anatomic crowns and contact areas that are wide facio-lingually and apico-coronally (Fig. 21).

The tooth morphology appears to be correlated with the soft tissue quality. The triangular tooth shape is associated with the scalloped and thin periodontium. The contact area is located in the coronal third of the crown underlining a long and thin papilla. The square anatomic crown shape combines with a thick and flat periodontium. The contact area is located at the middle third supporting a short and wide papilla.

Loss of interproximal tissue in the presence of a triangular tooth form will display a wider black triangle than in a situation when a square tooth is present (Fig. 21). In some cases when the adjacent teeth are to be restored the crown form can be modified prosthetically to compensate for partial interproximal tooth loss. The contact 
Fig. 1 (left) Intra oral photograph of implant in poor position angled labially and exiting the ridge too coronally

Fig. 2 (right) Restoration fabricated for implant in Figure 1; note length of restoration compared to adjacent teeth

Fig. 3 (left) Labial view of restoration for implant on Figure 1 note poor shape and form of the tooth dictated by poor implant position

Fig. 4 (right) Restoration of implants must satisfy objective and subjective aesthetic criteria. There should be sufficient inter-radicular space for placement of the implant and sufficien inter-tooth distance for fabrication of an aesthetically pleasing restoration

Fig. 5 (left) Note ceramics of right central incisor matches that of the left central incisor, however reconstruction of the deficient hard tissue has not been achieved, not satisfy objective criteria of aesthetics

Fig. 6 (right) Implant restoration on left lateral incisor in harmony with the existing hard and soft tissue

Fig. 7 (left) Wound healing following extraction of a tooth can of the inter-dental papilla

Fig. 8 (right) A perfect indication for a dental implant is nonpreparation of the adjacent teeth

\section{Fig. 9 (left) Low smile line}

Fig. 10 (right) Average smile line

Fig. 11 (left) High smile line. The colour and contour of the restorations and associated hard and soft tissues becomes very visible to the observer

Fig. 12 (right) The right lateral incisor has been treatment planned for an implant restoration. The level contra lateral tooth resulting in a restoration that does result in apical and palatal migration of the soft tissues mimic that of the
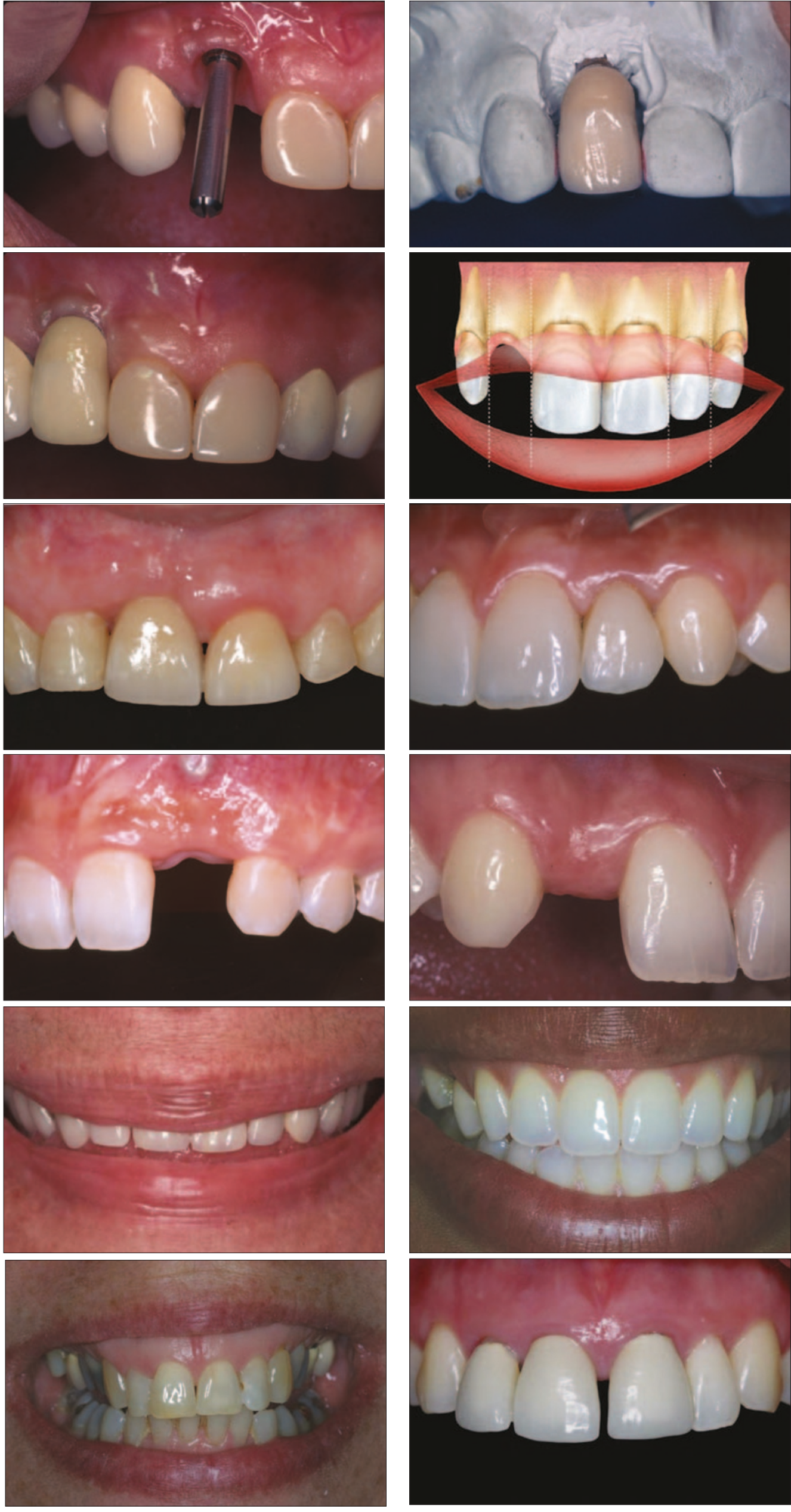

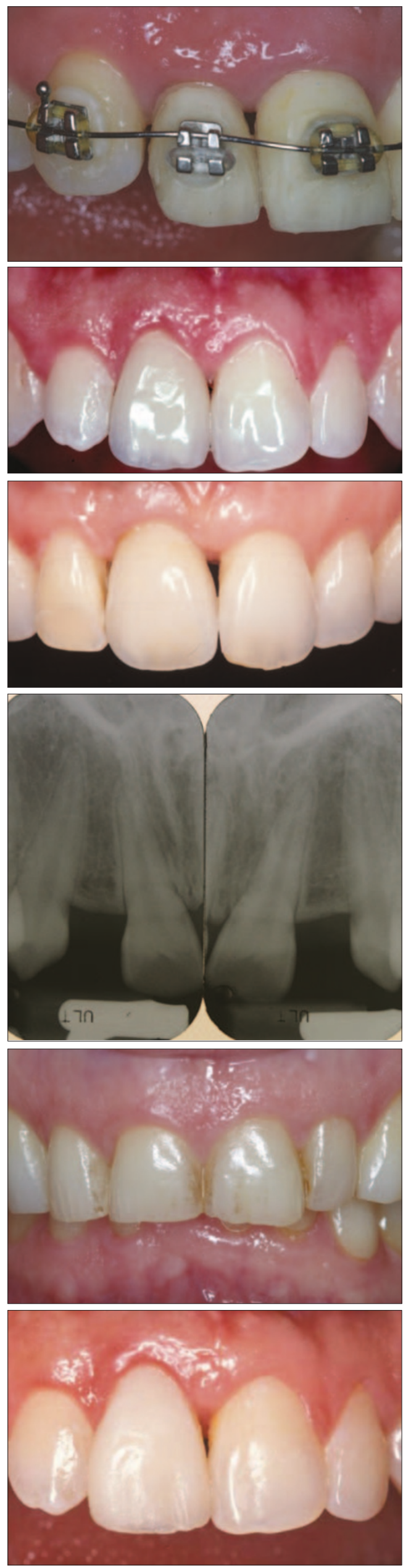
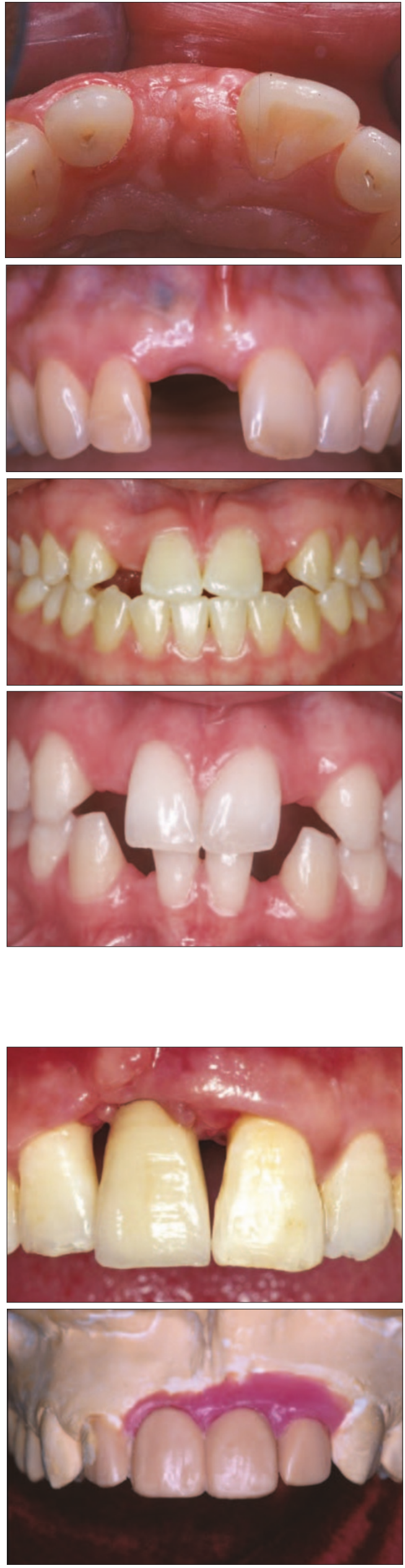

Fig. 13 (left) Immediate extraction of the right lateral incisor would result in apical migration of the soft tissue. Orthodontic extrusion will allow the clinician to position the tissue more coronally so that on extraction there is a margin of error

Fig. 14 (right) The mesio-distal width of the tooth requiring replacement must equal that of the contra lateral tooth

Fig. 15 (left) Implant restoration replacing the right central incisor

Fig. 16 (right) Excessive mesiodistal space in the region of the tooth requiring an implant restoration

Fig. 17 (left) Implant restoration in the region of the right central incisor. Note absence of interdental papilla as a result of inadequate support of the soft tissue by the restoration

Fig. 18 (right) Clinical presentation of patient with congenitally missing maxillary lateral incisors post orthodontic treatment

Fig. 19 (left) Radiograph of patient in Figure 18 revealing that there is insufficient inter radicular space for implants

Fig. 20 (right) Biotype 1 periodontium; note thin and scalloped tissue

Fig. 21 (left) Biotype 2

periodontium, not thick and flat tissues

Fig. 22 (right) Loss of interproximal soft tissue in the presence of a triangular tooth form can result in unsightly black triangles

Fig. 23 (left) Overcontour of the implant provisional restoration as it emerges from the free gingival margin can result in apical migration of the soft tissues

Fig. 24 (right) A diagnostic wax up can highlight the deficiency of the hard and soft tissue and can indicate to the surgeon how much augmentation is required 
area of the prosthetic tooth is positioned more cervically reducing the volume of the interdental space.

The presenting tooth shape will also influence the implant restoration shape. The implant restoration should mimic its contra lateral natural tooth coronal to the free gingival margin. However, apical to the free gingival margin, the implant restoration will not be an anatomic replica. A delicate balance must be developed that provides adequate support of the gingival architecture yet does not provide excessive pressure. Ideally the facial contour should be slightly flatter than the contra lateral natural tooth to minimise apical displacement of the free gingival margin after insertion (Fig. 23). ${ }^{14}$

\section{Bony anatomy of the implant site}

For successful aesthetic restoration of implants, the bony housing must have a three dimensional configuration that permits placement of an implant in a restoratively ideal position. ${ }^{15}$ If the bony anatomy is inadequate, a bone grafting procedure may be required to enhance the site. When these situations are encountered the patient must be made to understand that a successful outcome will involve replacing more than just a 'missing tooth'. The patient must also understand that the missing hard and soft tissue architecture will need to be rebuilt so that optimum aesthetics can be achieved.

The definitive implant restoration needs to be surrounded by a hard and soft tissue environment which is in harmony with the surrounding dentition. It is not only the amount of bone and soft tissue present prior to implant surgery but the precision of surgical execution which leads to an overall favorable outcome. Several key analyses need to be performed prior to commencing with implant placement. A diagnostic wax up highlighting tissue deficiencies and final tooth positioning can assist in the planning process (Fig. 24).

Facio-lingual ridge anatomy should be evaluated to determine if there is sufficient crest width to house the implant. Deficient alveolar crest width will require a bone augmentation procedure to allow the implant to be placed in the ideal position (Fig. 25). Clinical sounding techniques or sophisticated radiographic techniques such as tomograms or CT scans can assist in diagnosing deficiencies in this dimension (Figs 26 and 28).

Mesio-distal space should be equal to that of the contra lateral tooth; excess or deficiencies in this dimension need to be addressed through orthodontics, enameloplasty or restoration either prior to or after implant placement.

The most critical dimension remains the apicocoronal dimension; deficiencies in this dimension can result from periodontal disease, trauma, atrophy and infection. Vertical grafting is complex and the site may require several surgeries to achieve an optimal configuration. The most efficient method to evaluate this dimension is through the use of a diagnostic template highlighting the proposed gingival margin of the implant restoration.

Two anatomic structures are important in determining predictability of soft tissues after implant placement. The first is the height and thickness of the facial bony wall and the second is the bone height of the alveolar crest in the interproximal areas.

\section{Height and thickness of facial bony wall}

The position of the osseous crest is an important predictor for gingival levels. Kois, ${ }^{16}$ in a survey of 100 patients, classified patients as having high, normal or low crests. This was based on the vertical distance of the osseous crest to the free gingival margin. The greater the distance from the osseous crest to the free gingival margin the greater the risk of tissue loss after an invasive procedure. Kois proposed that if the total vertical distance of the total dentogingival complex on the mid facial aspect is $3 \mathrm{~mm}$, a slight apical loss of tissue up to $1 \mathrm{~mm}$ is anticipated after extraction and immediate implant placement. Greater or lesser than $3 \mathrm{~mm}$ indicates the change will be relatively negligible to more than $1 \mathrm{~mm}$. Measuring the distance from the free gingival margin to the osseous crest prior to extraction is an important diagnostic predictor of the anticipated final position of the free gingival margin.

\section{Height of bony crest in the interproximal area}

The interproximal bony crest plays a critical role in the presence or absence of peri-implant papillae. A clinical study around teeth ${ }^{12}$ measured the distance from the interproximal contact to the vertical height of bone and observed how frequently the interproximal space would be filled completely by soft tissue. When the contact point to the bone was 3-5 mm, papilla always filled the space. When the distance was $6 \mathrm{~mm}$ papilla was absent $45 \%$ of the time and with a distance of $7 \mathrm{~mm}$, papilla did not fill the space $75 \%$ of the time. A difference of $1-2 \mathrm{~mm}$ is significant in obtaining soft tissue aesthetics. This has been confirmed with implant supported restorations. ${ }^{17}$ Kan et al. ${ }^{18}$ have also shown that the height of peri-implant papillae in single tooth gaps is independent of the proximal bone level next to the implant but is dependent on the interproximal bone height of the adjacent teeth. From a diagnostic perspective sounding from the tip of the papilla to the interproximal bone crest of the adjacent tooth would be an important predictor (Figs 29-30). If this distance is $5 \mathrm{~mm}$ or less there is an increased likelihood that the interproximal tissues will be predictably maintained following implant placement and restoration. If the distance is greater than $5 \mathrm{~mm}$ the papilla cannot be predictably maintained after surgical intervention (Figs 30, 31).

\section{Implant position}

Aesthetic implant placement is driven by both a restorative and biological philosophy. Aesthetically the implant should be placed to satisfy the parameters of contour so that the restoration is pleasing. Biologically it should be placed 
to allow maintenance of both hard and soft tissue architecture. If the tooth to be replaced has not yet been removed, several determinations should be made prior to the extraction. ${ }^{9,10}$ Immediately placing the implant after extraction helps to shorten the treatment time and may reduce the amount of ridge width reduction that accompanies tooth extraction. In addition, if bone deficiencies are present, orthodontic eruption of the tooth prior to extraction can help to increase the amount of hard and soft tissue in the future implant site. ${ }^{19-21}$

The need for precision in implant placement varies according to each individual case. For example, in the edentulous mandible there is need for precision only in the facio-lingual direction. The need for precision increases in the partially edentulous jaws according to the teeth treated and the positions of the neighbouring and opposing teeth. The most challenging is the anterior maxilla where a malposition of less than a $1 \mathrm{~mm}$ can jeopardise the overall treatment outcome.

In most situations involving a single anterior implant restoration, the aesthetic considerations are more important than functional considerations. As such axial loading is not as critical as it is with posterior implant restorations. Implant position is critical to the final aesthetic outcome and needs to be considered in all three dimensions and in relation to the adjacent teeth. Misalignment of the implant in the prosthetic space can have adverse aesthetic consequences.

\section{Apico-coronal placement}

Apicocoronal positioning appears to be the most critical aspect. Deficient tissue in this dimension can result from several factors. This type of tissue needs to be addressed during treatment planning. Because of the complexity of vertical hard and soft tissue grafting these patients are placed in a high risk group.

Most often tooth loss is followed by bone loss of minor or major importance. It is necessary to evaluate the discrepancy between the bone level at the proposed implant site and the level at the adjacent teeth. Too large a difference represents a risk to both periodontal and peri-implant tissue health. Facing this, the surgeon should consider reconstructing the ridge prior to implant placement.

The apico-coronal positioning of the implant is the vertical discrepancy between the occlusal surface of the implant and the peaks of the bony septa proximal to the adjacent teeth, the most pleasing aesthetic result occurs when this discrepancy is minimal.

To obtain appropriate apicocoronal positioning of the implant, a diagnostic wax up needs to be completed and from this a surgical guide is made. The emergence profile and the shape of the restoration are reproduced on the guide to verify the implant positioning on placement.

A maxillary central incisor measures on average 7-8 $\mathrm{mm}$ mesiodistally and $6 \mathrm{~mm}$ faciolingually at the emergence from the soft tissue. A $4.0 \mathrm{~mm}$ the implant needs to be placed 3-4 $\mathrm{mm}$ apical to the gingival margin of the contra lateral tooth to allow the restoration to emerge with a natural profile. A vertical distance of 3-4 $\mathrm{mm}$ is needed for gradual transition from the 4 $\mathrm{mm}$ diameter of the implant platform to the 7-8 $\mathrm{mm}$ dimension at the gingival margin. If a lateral incisor is being replaced the implant would not have to be placed so apically since the average diameter of the crown at the gingival margin is $5 \mathrm{~mm}$ and less room is required for transition (Figs 33-37).

There are also situations in which there is excess tissue height and these require attention as well. In these types of patients a bone scalloping procedure is required to allow placement of the implant shoulder in a subgingival position, once again the most efficient way to examine this is through a surgical guide highlighting the proposed gingival margin.

Errors in apico-coronal implant placement can have serious aesthetic and biomechanical implications. An implant placed too coronally will not allow adequate transition from the head of the implant to the point where the restoration exits from the free gingival margin. The restoration will look short in comparison to the contra lateral tooth. The only prosthetic 'bailout' for this type of situation is to provide a ridgelapped restoration with contours that are pleasing to the observer's eye (Figs 38-39).

Problems can also result when implants are placed too apical. Clinically if an implant is placed too apically with excessive countersinking procedures an unnecessary amount of bone loss will occur (Fig. 40). Because this bone loss takes place circumferentially it will affect not only the proximal bone structure but also the height of the facial bone wall and can lead to undesirable soft tissue contours. ${ }^{22}$

A practical problem in placing an implant too deep is access for instrumentation. Making an impression of a deeply place implant can be a difficult experience (Fig. 41). The soft tissue tends to collapse, there is tissue impingement when trying to locate the head of the implant and seating is difficult to evaluate. This is specific to external hex systems.

If an implant is placed too deep a screw retained restoration is the treatment of choice. The literature shows that removing all the cement when an implant is placed so deep can prove to be a difficult endeavor. Agar et al. ${ }^{23}$ found that when six experienced investigators were asked to remove cement there was a surprising amount of cement left behind, these can lead to serious soft tissue complications.

\section{Mesio-distal placement}

Improper mesiodistal positioning of implants can also have a substantial effect on the generation of interproximal papillary support as well as on the osseous crest of the adjacent tooth. An implant should be placed 1.5-2 $\mathrm{mm}$ from an adjacent tooth placement too close to the adjacent tooth can cause resorption of the interproximal alveolar crest to the level of that on the implant. ${ }^{24}$ With this resorption comes a reduction in 
Fig. 25 (left) A deficient alveolar crest will not allow the implant to be placed in an ideal position. There will be insufficient bony housing to accommodate the fixture

Fig. 26 (right) Clinical slide of inadequate bucco-lingual width for implant placement

Fig. 27 (left) Bone augmentation of the site pictured in Figure 26

Fig. 28 (right) Ideal implant placement; note adequate ridge contours

Fig. 29 (left) If attachment of adjacent teeth is deficient it is unlikely that the interdental papilla will be maintained

Fig. 30 (right) Black triangles are likely to result between the implant restoration and adjacent teeth

Fig. 31 (left) Diagnostic sounding of the bone interproximally is a good clinical indicator in predicting post treatment papilla levels

Fig. 32 (right) Radiograph of Figure 31 , the right lateral incisor has been treatment planned for an implant restoration. Due to attachment loss on the adjacent canine it is unlikely that the interproximal papilla will be maintained

Fig. 33 (left) Ideal implant placement for central incisor. Implant should be placed $3-4 \mathrm{~mm}$ apical to the existing free gingival margin. Adequate room is required for transition from the head of the implant to the point where the restorations exits the free gingival margin. Note minimal bone discrepancy between the implant and the adjacent teeth

Fig. 34 (right) Laboratory slide depicting ideal implant placement and transition required
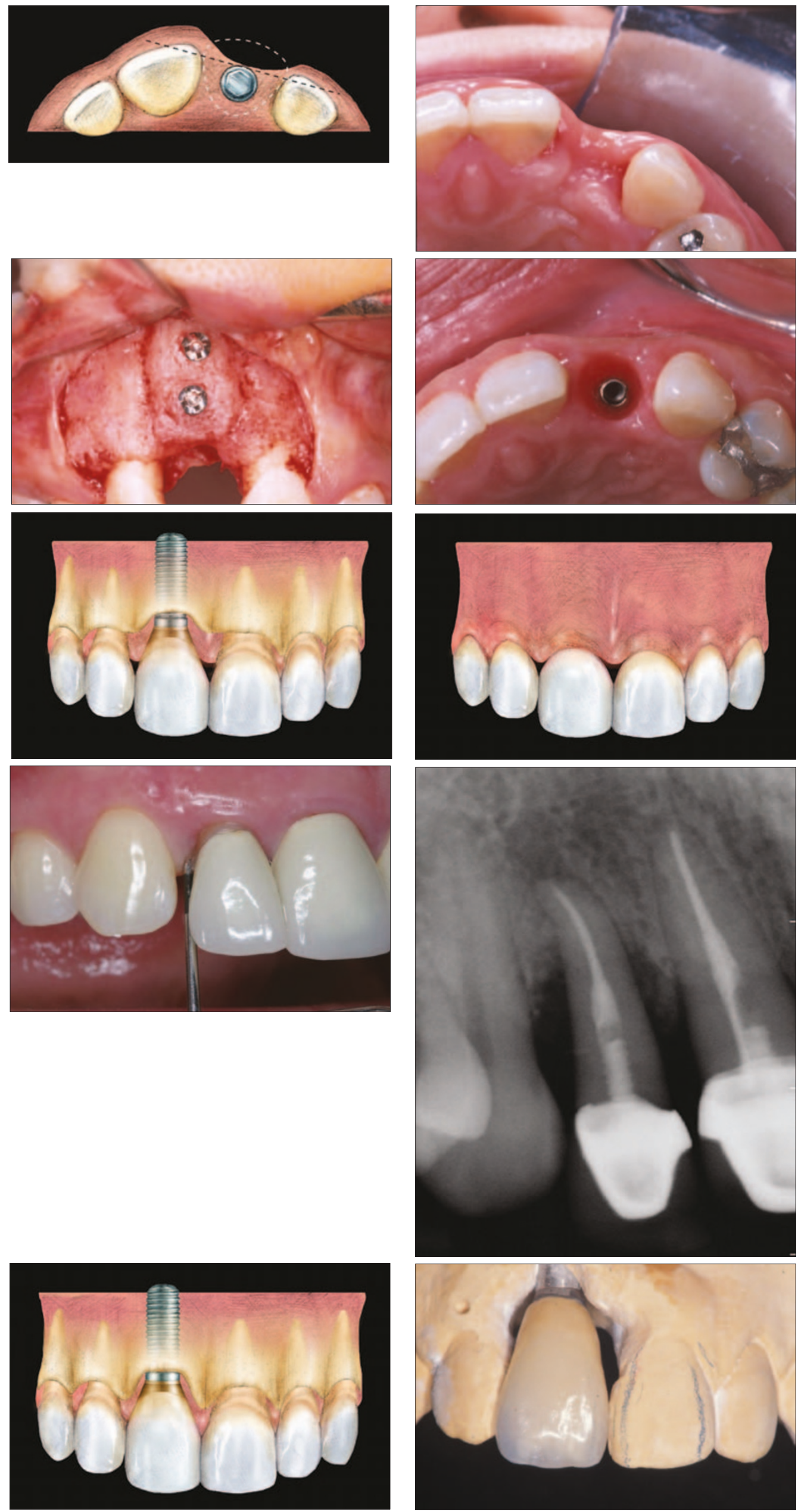

BRITISH DENTAL JOURNAL VOLUME 201 NO. 2 JUL 222006 

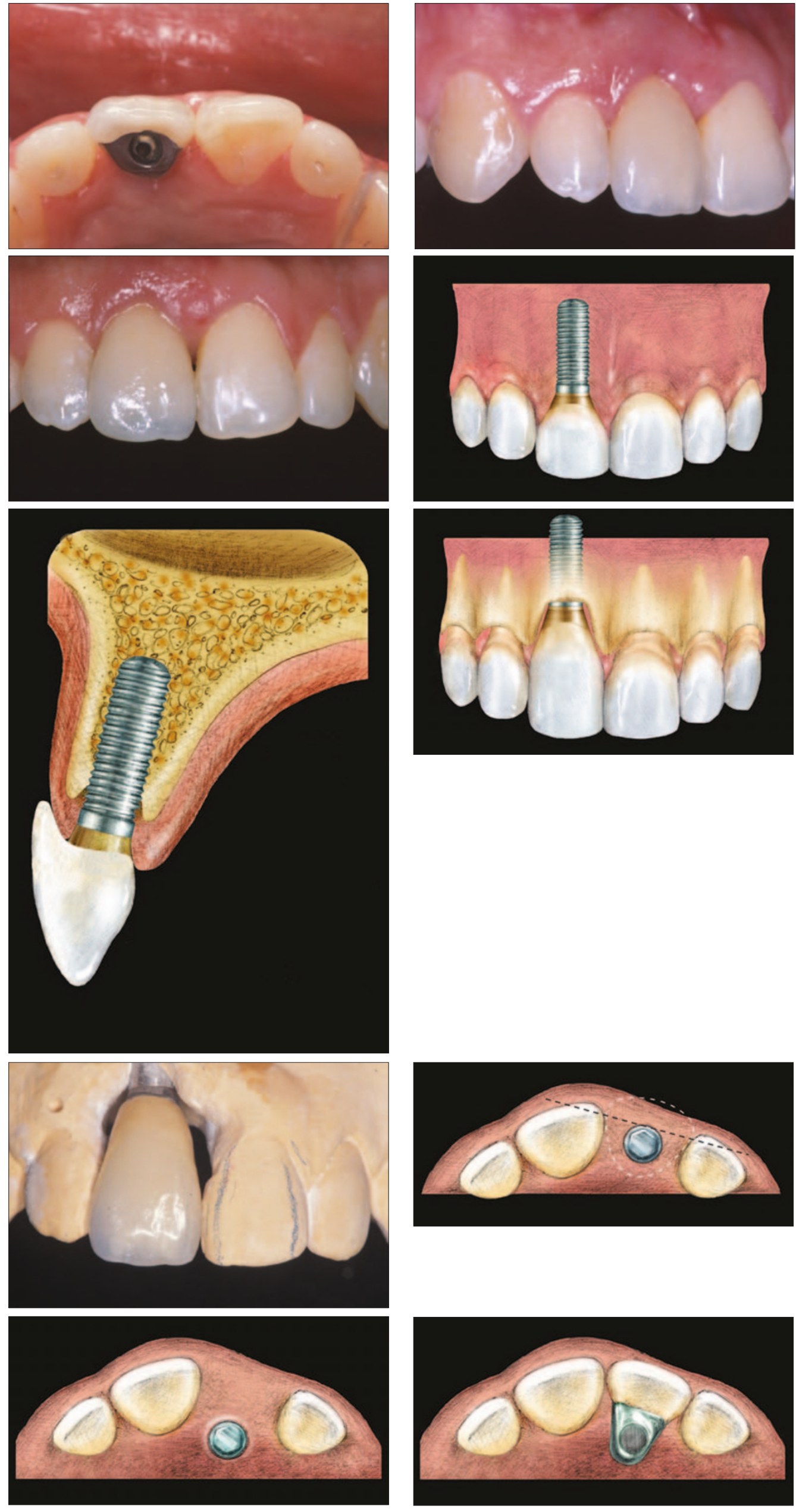

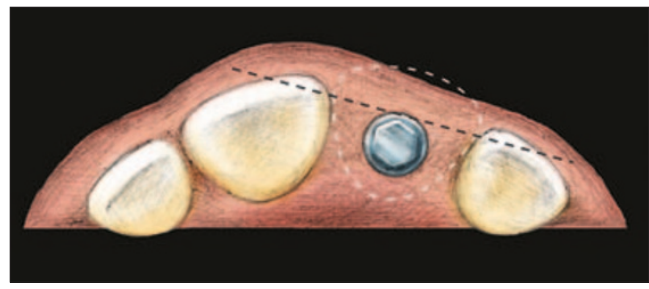

Fig. 35 (left) Occlusal view of implant restoration shown in Figure 34

Fig. 36 (right) Lateral view of implant restoration in Figure 34 showing fill of interproximal soft tissue
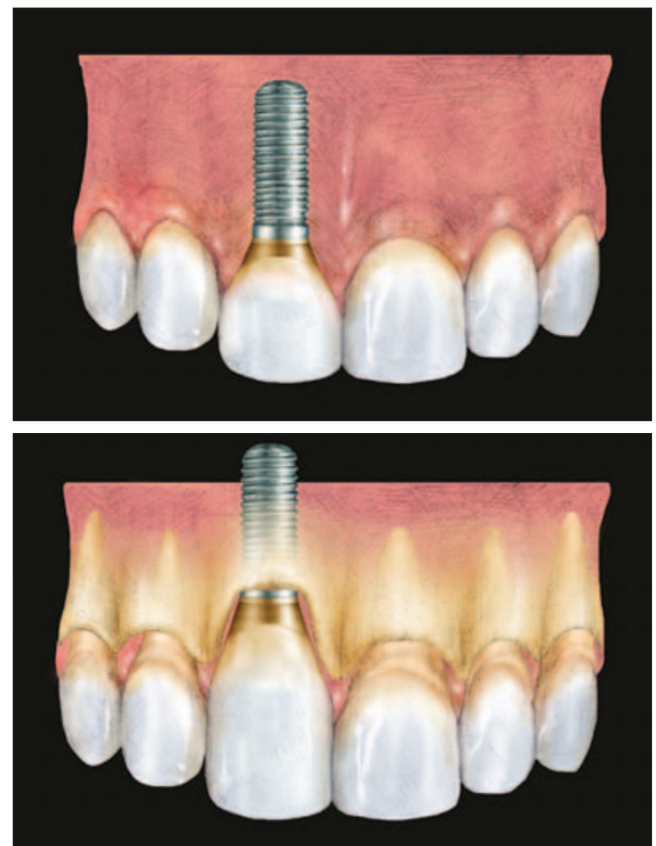

Fig. 37 (left) Facial view of implant restoration in Figure 34 showing aesthetic harmony

Fig. 38 (right) Placement of an implant too shallow will result in inadequate space for transition and a short restoration in length

Fig. 39 (left) A prosthetic bailout for too shallow implant placement is to ridge lap the restoration onto the tissues

Fig. 40 (right) Too deep an implant placement can result in biomechanical problems. Note level of the bone around implant in relation to the adjacent teeth. This deep a placement can result in fistula formation and constant post operative maintenance problems

Fig. 41 (left) Too deep an implant placement can result in soft tissue collapse and difficulty in instrumentation

Fig. 42 (right) Ideal implant placement should be palatal to an imaginary line that outlines the curvature of the teeth. (Modified from Parel S M, Sullivan D Y) ${ }^{28}$

Fig. 43 (left) Too palatal an implant placement

Fig. 44 (right) The restoration for the implant placement in Figure 43 would require an excessive facial cantilever 
papillary height. Restorative problems exist as well. Poor embrasure form and emergence profile will result in a restoration with a long contact zone and compromised clinical outcomes.

The loss of crest height on adjacent teeth is caused by bone saucerisation routinely found around the implant shoulder in implants. This has two dimensions: a horizontal and vertical. Radiographs only demonstrate the horizontal aspect of bone saucerisation; the proximal bone saucerisation measures 1-1.5 $\mathrm{mm}$ from the implant surface. This distance needs to be respected on implant placement to prevent vertical bone loss on adjacent teeth. ${ }^{25}$

\section{Facio-lingual placement}

- The crest width needs to be examined to determine the presence or absence of bone atrophy.

- Placement will vary depending on the mechanism of retention of the final restoration (screw retained vs. cement retained).

- Deficient alveolar crest width may require augmentation so that the implant can be positioned in the correct facio-lingual position.

Computerised tomographic scan techniques are useful in assisting to determine width. The amount of bone available should be at least 1 $\mathrm{mm}$ greater than the implant diameter on each side. Hence a $4 \mathrm{~mm}$ diameter implant would require $6 \mathrm{~mm}$ of bone. The single implant placed in the maxillary anterior region should be situated palatal to an imaginary line that outlines the curve of the arch formed by the facial surfaces of the adjacent teeth (Fig. 42). ${ }^{26}$

Implants placed too palatal complicate development of hygienic contours. Biomechanical complications can also arise as a result of cantilever forces on the screw joint of external hex systems (Figs 43-45). Implants are often mistakenly placed too facial. This error results in excessive resorption of the supporting osseous structure resulting in a restoration that will appear long in comparison to the contra-lateral tooth. Placement of a restoration such as this in the aesthetic zone is certainly unlikely to meet the patient's desires (Figs 46-47).

\section{Considerations for multiple implants}

Patients with extended edentulous spaces present greater anatomic and aesthetic challenges making it even more difficult to obtain an aesthetic result with certainty. Following extraction and wound healing of two adjacent teeth, the ensuing apical and facio-lingual resorption results in an edentulous segment which is flattened. The same diagnostic considerations need to be addressed as when looking at single tooth edentulous sites. The aim prior to implant placement is to have a threedimensional configuration of hard and soft tissue which will allow placement of implants in an ideal position. The placement of two missing central incisors poses an additional challenge. Following surgical placement an additional peri-implant bone remodelling takes place. In the frontal plane two processes occur: one between the implant and the adjacent natural tooth and one between the two adjacent implants. On the tooth implant side the predictability of the interdental papilla is governed by the height of the interproximal bone crest of the tooth. If this height is favourable there is good certainty that the interdental papilla will be maintained following implant placement. The bone crest between the two implants is likely to undergo further resorption in an apical direction; this is accompanied by a loss of interimplant soft tissue which in the case of multiple edentulous sites will result in black triangles between the adjacent restorations.

Many clinicians have sought after the ideal implant distance required to maintain the interdental papilla. Tarnow and colleagues ${ }^{11}$ performed a radiographic study to address this clinical problem. Radiographic measurements were taken at a minimum of one or three years after implant exposure. All radiographs were taken with a paralleling technique.

Radiographs were computer scanned imaged and magnified for measurement. The following measurements were taken:

1. Lateral distance from the crest of the interimplant bone to the implants

2. Vertical crestal bone loss

3. Distance between the implants at the implant/ abutment interface.

When implants were placed too close together the bone remodelling overlapped to a great degree and consequently resulted in loss of vertical bone height which subsequently had soft tissue implications.

When implants were placed $3 \mathrm{~mm}$ and greater, lateral bone loss from the adjacent implants did not overlap with minimal resultant crestal bone loss. They concluded that it is more difficult to create or maintain papilla between two adjacent implants than it is between an implant and a natural tooth. Their recommendation was that when two implants are placed adjacent to each other in the aesthetic zone, a minimum of $3 \mathrm{~mm}$ of bone should be retained between them at the implant/ abutment level (Figs 48-49). This particular study addressed bone loss between the implants. It should be remembered that the bone saucerisation has two dimensions - a horizontal and a vertical. Radiographs only demonstrate the horizontal aspect of bone saucerisation. Bone loss occurs circumferentially around the implant and when two implants are placed adjacent to each other facial bone loss also occurs (Figs 50-51). This has implications in terms of stability of the facial gingival margin. If the implants are placed too far forward there will be less facial bone and this will ultimately result in apical migration of the free gingival margin (Fig. 52). Placement of adjacent implants is also critical for restorative contours; placing implants too close together makes it difficult for the laboratory technician to fabricate restorations with pleasing aesthetic contours.

Tarnow and colleagues ${ }^{27}$ also performed a study to determine the height of the soft tis- 
sue to the crest of the bone between two adjacent implants. This was done independent of the location of the contact point. They looked at 136 inter-implant papillary heights in 33 patients by eight examiners. A standardised periodontal probe was used and placed from the height of the papilla to the crest of the bone. What they found was that the mean height of papilla between two adjacent implants was 3.4 $\mathrm{mm}$ with a range of 1-7 $\mathrm{mm}$.

Although this was a retrospective study and there were many variables such as operator, implant type, placement and so forth it did give us information that soft tissue between two adjacent implants in the aesthetic zone is not a predictable procedure, and when treatment planning, the patient must be aware of this or alterations must be made in the treatment plan to provide an aesthetic result.

Recreating interdental papilla between two adjacent implants is a formidable task. Restoratively, clinicians alter the position of the contact point to give the illusion of papilla. The thin spicule of bone remaining between the implants may be sufficient to maintain the papilla during the first few years of the restorations service. However, there are no clinical studies with long term results presented to date to illustrate the predictability of papilla between two adjacent implants.

Another clinically challenging situation is replacement of a maxillary canine and adjacent lateral incisor. This becomes clinically more challenging because the edentulous space is smaller and the inter-implant soft tissue tends to be less voluminous. Consideration in this instance should be given to placement of a single implant in the canine region and cantilevering a lateral incisor from it. Placement of the implant should follow all the principles discussed previously in the article.

Replacement of several missing teeth with implants allow for the use of fixed partial dentures and the opportunity to use ovate pontics to help support the soft tissue and give an illusion of papillae. The authors have encountered many situations where one implant per tooth philosophy is espoused to. This can be particularly detrimental in the aesthetic zone. The literature is quite clear that maintaining papillae between implants is not predictable. Even with the advent of scalloped type implants there are no long term studies showing papilla maintenance. For an aesthetic outcome it is more predictable to place implants away from each other so that the intervening soft tissue can be sculpted to give the illusion of papilla. One common error often published in the literature is placement of four implants to replace lateral incisor to lateral incisor. This philosophy of implant placement will not yield an aesthetic outcome (Fig. 55). Placement of two implants in both lateral incisor regions and fabrication of a fixed partial denture sculpting the intervening tissue with ovate pontics is likely to produce an illusion of papilla which will be more pleasing to the observer's eye (Fig. 56). This placement philosophy can also be reserved for extended edentulous spans where aesthetics is of paramount importance (Figs 57-58). Placement of implants in multiple edentulous spaces must follow the same principles as for single tooth situations; placement must follow appropriate diagnosis and treatment planning, which includes a diagnostic wax up and fabrication of a surgical guide to facilitate implant placement. If these techniques are not followed it is all too easy to easy to find implants in the wrong position where prosthetic strategies have to be used to satisfy the patient's demand for aesthetics. In situations like these patient expectations are unlikely to be met (Fig. 59-61).

\section{CONCLUSION}

When a patient has a missing anterior tooth and desires replacement, a decision must be made by the dentist and patient as to the method of replacement. Common choices would include a conventional fixed partial denture, a resin bonded fixed partial denture or an implant borne restoration. Each has its advantages and disadvantages. The conventional fixed partial denture has the advantages of being an established treatment procedure, of having predictable aesthetics, and being expedient. It has the disadvantage of requiring preparation of adjacent teeth and potential risk for periodontal and pulpal tissue. The resin bonded partial denture has the advantages of preserving tooth structure, having predictable aesthetics and reduced cost. It has the disadvantages of being technique sensitive for the dentist and technician and often losing retention which may lead to decay. Implants used to replace missing teeth in the aesthetic zone have many advantages from preservation of unrestored adjacent teeth, halting the resorption of edentulous spaces and providing support. However, at present it has the disadvantages of long treatment time, requiring a provisional restoration during implant integration, requiring surgical placement of the implant, requiring surgical uncovering of the implant, requiring a provisional after the implant is uncovered and having a higher cost. Much effort is being directed at shortening the treatment time and making delivery of the service more time efficient. Immediately loading implants is one direction that many researchers and clinicians are taking. However, the parameters to when immediately loading implants is possible have not been established and until that time, immediately loading implants must be made on an individual and case by case basis, taking into account all the factors that affect loading of the initially non osseointegrated implants.

Even with all the disadvantages listed, the implant supported single tooth restoration can be successfully executed when all the factors discussed in this article are addressed. When one or more of the adjacent teeth are unrestored or in need of only a minor restoration, the single tooth implant should be considered the restoration of choice. 
Fig. 45 (left) Profile of restoration in Figure 44 illustrating a

biomechanical and hygienic compromise

Fig. 46 (right) Too facial an implant placement

Fig. 47 (left) Too facial an implant placement will result in facial bone resorption and apical migration of the soft tissue. The resulting restoration will appear long in comparison to the contra lateral tooth

Fig. 48 (right) When implants are placed $3 \mathrm{~mm}$ and greater apart the bone loss from the adjacent implants does not overlap resulting in minimal crestal bone loss. (Modified from Tarnow et al..) ${ }^{11}$

Fig. 49 (left) When implants are placed too close together, bone loss from adjacent implants overlaps resulting in additional loss of the crestal bone. (Modified from Tarnow et al.."1)

Fig. 50 (right) Bone loss is

circumferential around the implants. When implants are placed too close together the vertical and horizonta components of bone loss compromise the peak of the interproximal bone and thus the resulting soft tissues

Fig. 51 (left) Ideal implant theoretically will maintain the interproximal peak of bone, however there are no long term studies to support this

Fig. 52 (right) If implants are place too facial this will compromise the thickness of the facial bony plate which can eventually resorb. This will result in apical migration of the soft tissue

Fig. 53 (left) Ideal implant placement will allow fabrication of restorations with ideal contours

Fig. 54 (right) Implants placed too close together will result in compromised restorative contour

Fig. 55 (left) Placement of adjacent implants compromises the inter implant peak of bone resulting in resorption and soft tissue loss

Fig. 56 (right) It is easier to develop illusion of papilla between an implant and an adjacent pontic. Implant placement philosophy should take advantage of such techniques
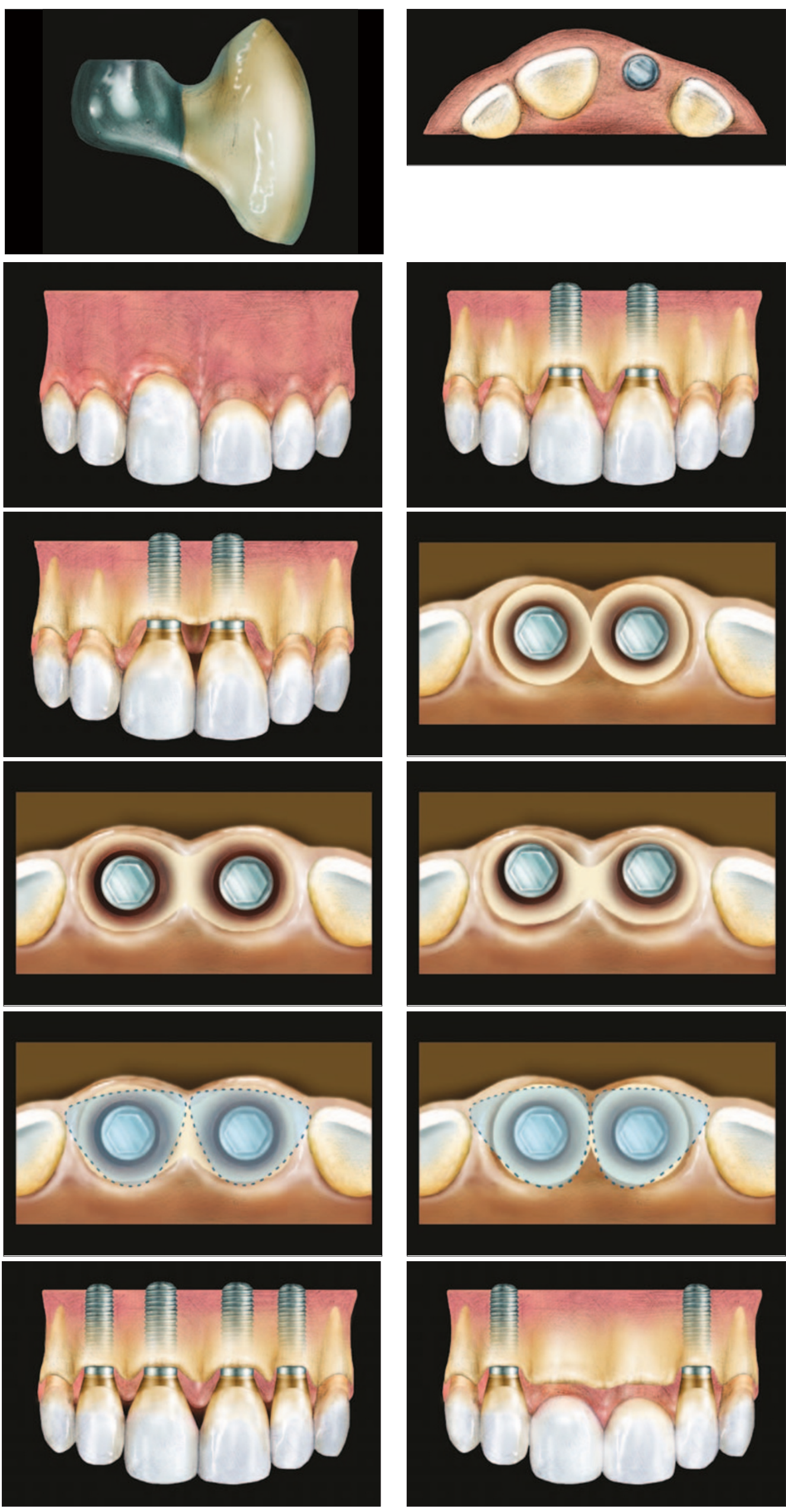

BRITISH DENTAL JOURNAL VOLUME 201 NO. 2 JUL 222006 

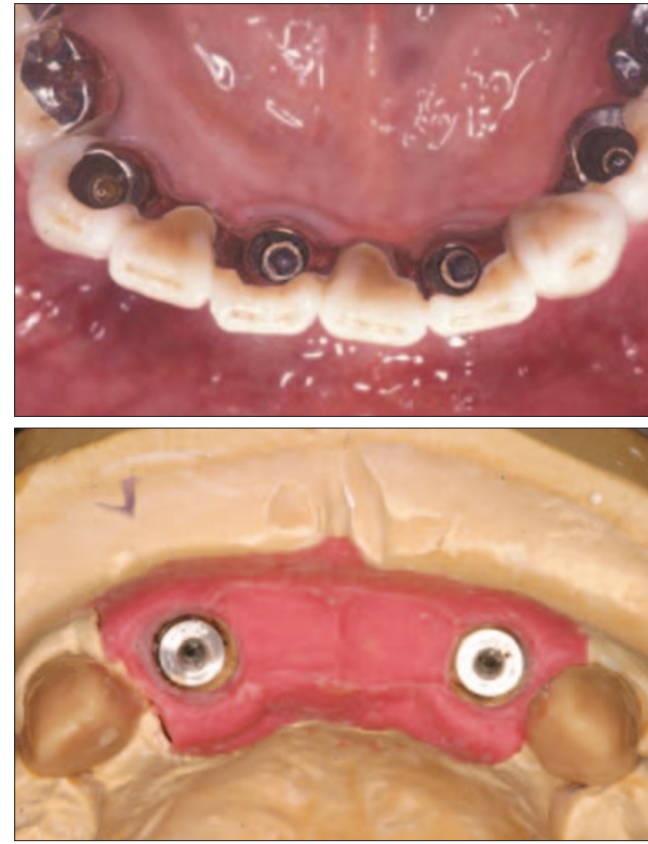

1. Sullivan R M. Perspective on aesthetics in implant dentistry. Compendium 2001; 22: 685-692.

2. Adell R, Eriksson B U, Branemark PI, Jemt T. A long term follow up study of osseointegrated implants in the treatment of totally edentulous jaws. Int J Oral Maxillofac Implants 1990: 5: 347-359.

3. Naert I, Quirynen M, van Steenberghe D, Darius P. A study of 589 consecutive implants supporting complete fixed prostheses. Part II: Prosthetic aspects. J Prosthet Dent 1992; 68: 949-956.

4. Belser U C. Esthetic checklist for the fixed prosthesis. Part II: Biscuit bake try in. In Scharer P, Rinn L A, Kopp F R (Eds). Esthetic guidelines for restorative dentistry. pp 188-192. Chicago: Quintessence, 1982.

5. Magne P, Belser U. Natural oral esthetics. In Bonded porcelain restorations in the anterior dentition. A biomimetic approach. pp 57-99. Chicago: Quintessence, 2002.

6. Tjan A H, Miller G D. The JG: Some aesthetic factors in a smile. J Prosthet Dent 1984; 51: 24-28.

7. Kois J C. Aesthetic extraction site development: The biological variables. Contemp Aest Rest Prac 1998; 2: 10-18.

8. Saadoun et al. Selection and ideal tri-dimensional implant position for soft tissue aesthetics. Prac Perio Aesthet Dent 1999; 11: 1063-1072.

9. Kois J C. Predictable single tooth peri-implant aesthetics: Five diagnostic keys. Compendium 2004; 25: 895-905.

10. Salama H, Salama M, Kelly J. The orthodontic-periodontal connection in implant site development. Prac Perio Aest Dent 1996: 8: 923-932.

11. Tarnow D P, Cho S C, Wallace SS. The effect of inter-implant distance on the height of inter-implant bone crest. J Periodonto/2000; 71: 546-549.

12. Tarnow D P, Magner A W, Fletcher P. The effect of distance from the contact point to the crest of bone on the presence or absence of the interproximal dental papilla. J Periodontol 1992; 63: 886-995.

13. Becker $W, O$ chsenbein $C$, Tibbetts L et al. Alveolar bone anatomic profiles as measured from dry skulls. Clinical ramifications. J Clin Periodontol 1997; 24: 727-731.

14. Phillips K, Kois J C. Aaesthetic peri-implant site development. The restorative connection. Dent Clin North Am 1998; 42: 57-70.

15. D'Addona A, Nowzari H. Intramembranous autogenous osseous transplants in aaesthetic treatment of alveolar atrophy. Periodonto/ 2000 2001; 27: 148-161.

16. Kois J C, Kan J Y. Predictable per-implant gingival aaesthetics. Surgical and prosthodontic rationales. Prac Perio Aest Dent 2001; 13: 691-698.

17. Choquet $V$, Adriaenssens $P$, Daelemans P et al. Clinical and radiographic evaluation of the papilla level adjacent
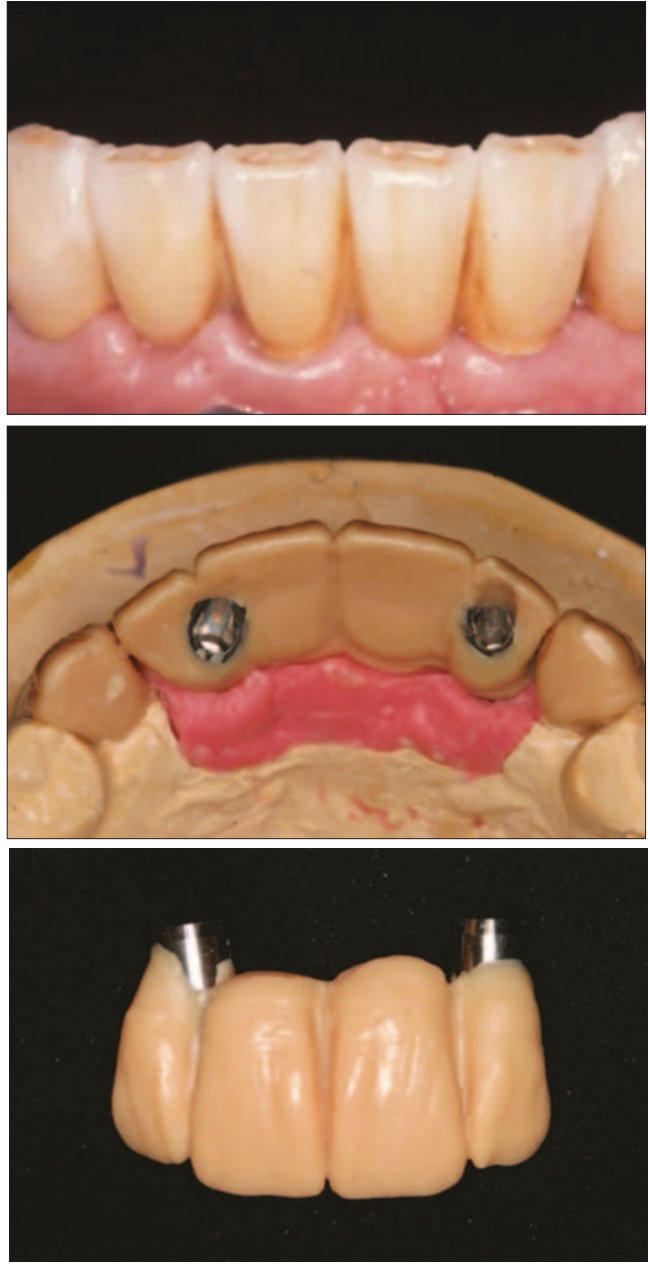

to single tooth implants. A retrospective study in the maxillary anterior region. J Periodonto/ 2001; 2: 1364-1371.

18. Kan J, Rungcharassaeng K, Umezu K, Kois J C. Dimensions of peri-implant mucosa. An evaluation of maxillary anterior single implants in humans. J Periodonto/ 2003; 4: 557-562.

19. Chee W W. Treatment planning and soft-tissue management for optimal implant aesthetics: a prosthodontic perspective. J Calif DentAssoc 2003; 31: 559-563.

20. Chee W W. Provisional restorations in soft tissue management around dental implants. Periodontol 2000 2001:27: 139-147.

21. Neale $D$, Chee W W L. Development of soft tissue emergence profile: A technique. J Prosthet Dent 1994; 71: 364-368.

22. Buser D, Martin W, Belser U C. Optimising aesthetics for implant restorations in the anterior maxilla: anatomical and surgical considerations. Int J Maxillofac Implants 2004; 19: suppl 43-61.

23. Agar J, Cameron S M, Hughbanks J C, Parker M H. Cement removal from restorations luted to titanium abutment with simulated subgingival margins. J Prosthet Dent 1997; 78: 43-47.

24. Thilander B, Odman J, Jemt T. Single implants in the upper incisor region and their relationship to the adjacent teeth. An 8 year follow up study. Clin Oral Implants Res 1999; 10 : 346-355.

25. Esposito M, Ekestubbe A, Grondahl K. Radiologica evaluation of marginal bone loss at tooth surfaces facing single Branemark implants. Clin Oral Implants Res 1993; 4: 151-157.

26. Chiche FA, Leriche M A. Multidisciplinary implant dentistry for improved aaaesthetics and function. Prac Perio Aest Dent 1998; 10: 177-186.

27. Tarnow D, Elian N, Fletcher P et al. Vertical distance from the crest of bone to the height of the interproximal papilla between adjacent implants. J Periodonto/ 2003; 74: 1785-178.

28. Parel S M, Sullivan D Y. Aesthetics and osseointegration.
Fig. 57 (left) Clinical slide showing Implant placement so that illusion of papilla can be developed

Fig. 58 (right) Facial view of slide in Figure 57

Fig. 59 (left) Laboratory slide illustrating facial placement of implants

Fig. 60 (right) Laboratory slide illustrating interproximal placement of implant in region of right lateral incisor. This is likely the occurrence of placement without a surgical guide

Fig. 61 Facial view of slide in Figure 60 depicting interproximal implant placement in the region of the right lateral incisor, this is likely to result in absence of interproximal soft tissue 